\title{
PAPER
}

\section{Field emission from two-dimensional GeAs}

To cite this article: Antonio Di Bartolomeo et al 2021 J. Phys. D: Appl. Phys. 54105302

View the article online for updates and enhancements.

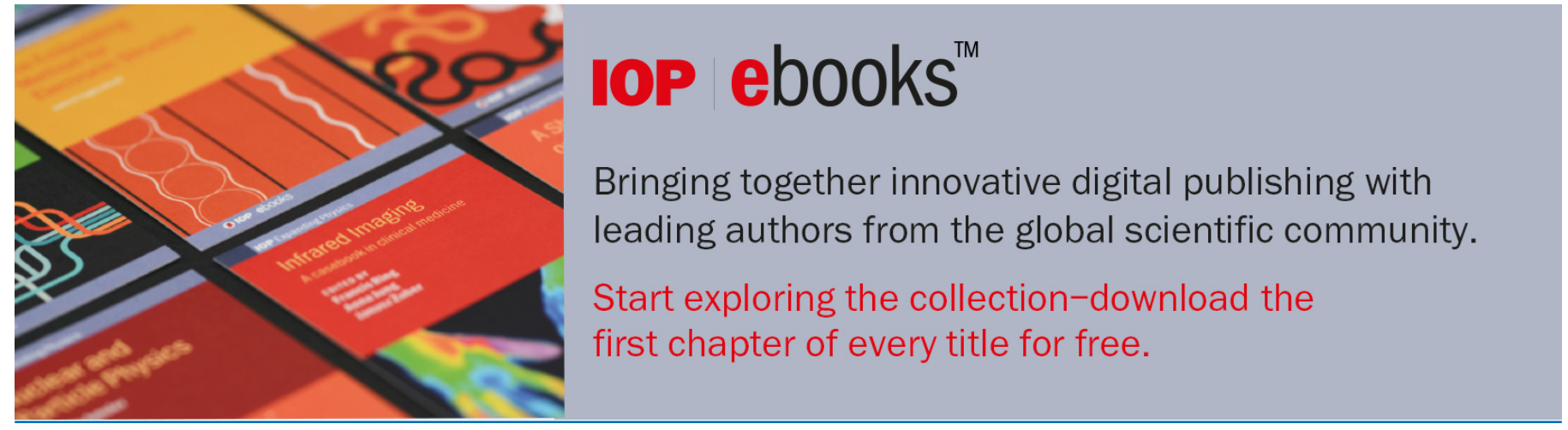

This content was downloaded from IP address 141.108 .253 .127 on $18 / 02 / 2021$ at $16: 32$ 


\title{
Field emission from two-dimensional GeAs
}

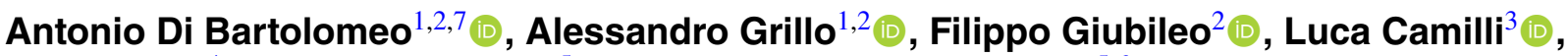 Jianbo Sun ${ }^{4}$, Daniele Capista ${ }^{5}$ and Maurizio Passacantando ${ }^{5,6}(\mathbb{B}$}

${ }^{1}$ Department of Physics 'E.R. Caianiello' and Interdepartmental Centre NanoMates, University of Salerno, via Giovanni Paolo II, Fisciano 84084, Italy

${ }^{2}$ CNR-SPIN Salerno, via Giovanni Paolo II, Fisciano 84084, Italy

${ }^{3}$ Dipartimento di Fisica, Università degli studi di Roma 'Tor Vergata', via della Ricerca Scientifica 1, Roma 00133, Italy

${ }^{4}$ Department of Physics, Technical University of Denmark, Ørsteds Plads, Kgs. Lyngby 2800, Denmark

${ }^{5}$ Department of Physical and Chemical Sciences, University of L'Aquila, via Vetoio, Coppito, L'Aquila 67100 , Italy

${ }^{6}$ CNR-SPIN L'Aquila, via Vetoio, Coppito, L'Aquila 67100, Italy

E-mail: adibartolomeo@unisa.it

Received 16 October 2020, revised 10 November 2020

Accepted for publication 20 November 2020

Published 22 December 2020

\begin{abstract}
GeAs is a layered material of the IV-V groups that is attracting growing attention for possible applications in electronic and optoelectronic devices. In this study, exfoliated multilayer GeAs nanoflakes are structurally characterized and used as the channel of back-gate field-effect transistors. It is shown that their gate-modulated p-type conduction is decreased by exposure to light or electron beam. Moreover, the observation of a field emission (FE) current demonstrates the suitability of GeAs nanoflakes as cold cathodes for electron emission and opens up new perspective applications of two-dimensional GeAs in vacuum electronics. FE occurs with a turn-on field of $\sim 80 \mathrm{~V} \mu \mathrm{m}^{-1}$ and attains a current density higher than $10 \mathrm{~A} \mathrm{~cm}^{-2}$, following the general Fowler-Nordheim model with high reproducibility.
\end{abstract}

Keywords: GeAs, 2D materials, field-effect transistor, field emission, electrical conductivity, anisotropy

(Some figures may appear in colour only in the online journal)

\section{Introduction}

Germanium arsenide (GeAs) has emerged as an interesting layered compound of IV-V groups with a crystal structure belonging to the centrosymmetric monoclinic $C 2 / m(12)$ space group and strong in-plane anisotropy $[1,2]$. In GeAs, every Ge atom is coordinated to three As atoms and another Ge atom, while every As atom is coordinated to three Ge atoms (figure 1(a)). Each GeAs monolayer is terminated by As atoms and interacts with neighboring layers by weak van der Waals forces. The small interlayer cohesion energy $\left(0.191 \mathrm{eV}\right.$ atom $\left.^{-1}\right)$ allows easy exfoliation. Ingots of GeAs

Author to whom any correspondence should be addressed. have been synthesized by combining $\mathrm{Ge}$ and As in vacuum at high temperature $(>1370 \mathrm{~K})$ [2] and have been exfoliated in liquid phase or mechanically to obtain multilayer or fewlayer nanosheets [3]. First-principles calculations and UVvisible absorption spectroscopy have demonstrated that GeAs nanosheets have a bandgap that increases significantly as the number of layers decreases, from $0.6 \mathrm{eV}$ for the bulk up to $2.1 \mathrm{eV}$ for the monolayer [3, 4]. Remarkably, while the monolayer has a direct bandgap, the multilayers are predicted to have a quasi-direct bandgap.

The electrical properties of multilayer GeAs nanosheets have been measured to reveal their two-dimensional (2D) carrier transport behavior [5] and anisotropic electrical conduction strongly affected by impurities [6]. Using multilayer GeAs field-effect transistors (FETs), it has been proved that the 
temperature-dependent conductivity can be described by the coexistence of variable range hopping among defect-induced bandgap states and band-like transport $[3,5,6]$. The carrier mobility in GeAs is higher along the zig-zag direction and typically in the range $0.1-10 \mathrm{~cm}^{2} \mathrm{~V}^{-1} \mathrm{~s}^{-1}$ [5-7]. The anisotropic crystal structure of GeAs leads also to highly anisotropic thermal conductivity [2], mechanical response [8] and optical properties [7]. Moreover, stable and large photocurrents with rapid on/off switching as well as linear dichroism and polarized photodetection indicate that GeAs is a promising material for high-performance optoelectronic nanodevices, particularly for polarization optical applications [3, 9]. GeAs nanosheets have also shown promising photoelectrochemical water splitting capability under visible light irradiation $[3,10]$.

In the present study, we characterize crystal structure and symmetry of mechanically exfoliated multilayer GeAs nanosheets. FETs are obtained by contacting GeAs nanoflakes with $\mathrm{Au}$ electrodes and/or metallic tips. We investigate the field emission (FE) properties of GeAs nanoflakes, taking advantage from a nanotip inside a scanning electron microscope (SEM) chamber that is used as the anode for local FE characterization $[11,12]$.

FE is the extraction of electrons from a semiconducting or metallic material under the application of an electric field. As a macroscopic manifestation of a quantum effect, FE offers significant scientific interests in material science and is exploited in many applications such as electron microscopy, electron spectroscopy, e-beam lithography as well as in vacuum electronics for nanoscale FE transistors, displays and microwave generation or for $\mathrm{x}$-ray tubes [13-19]. The externally applied electric field reduces the barrier for electron escape from the material to vacuum. FE is favored from electrically and thermally highly conducting materials with low work function and nanometer rough surface that give rise to local field enhancement. Hence, the intrinsic doping, the sharp edge and the low electron affinity below $4 \mathrm{eV}$ of GeAs nanosheets are beneficial for FE. Moreover, the GeAs electron affinity, which results in a low tunneling barrier, decreases with the number of layers, being only $3.17 \mathrm{eV}$ for the bilayer and $2.78 \mathrm{eV}$ for the monolayer [3]. Despite that, we note that FE from 2D GeAs has not been reported so far.

In this work, we show that a high and reproducible FE current can be extracted from the edge of GeAs nanoflakes.

\section{Materials and methods}

Ultrathin GeAs flakes were mechanically exfoliated from bulk GeAs single crystals using a standard adhesive tape method [20]. The nanoflakes were exfoliated onto degenerately ptype doped silicon substrates, covered by $300 \mathrm{~nm}$ thick $\mathrm{SiO}_{2}$. The $\mathrm{SiO}_{2} / \mathrm{Si}$ substrate was endowed with patterned markers of $5 \mathrm{~nm} \mathrm{Ni} / 50 \mathrm{~nm} \mathrm{Au}$, which were previously defined by photolithography and lift-off. Sometimes, the transferred flakes ended up partially over the $\mathrm{SiO}_{2}$ layer and partially over an Au marker establishing an electrical contact with it. The SEM images of two typical flakes are shown in figures 1(b) and (c). In particular, figure 1(b) displays a flake with minimal overlap
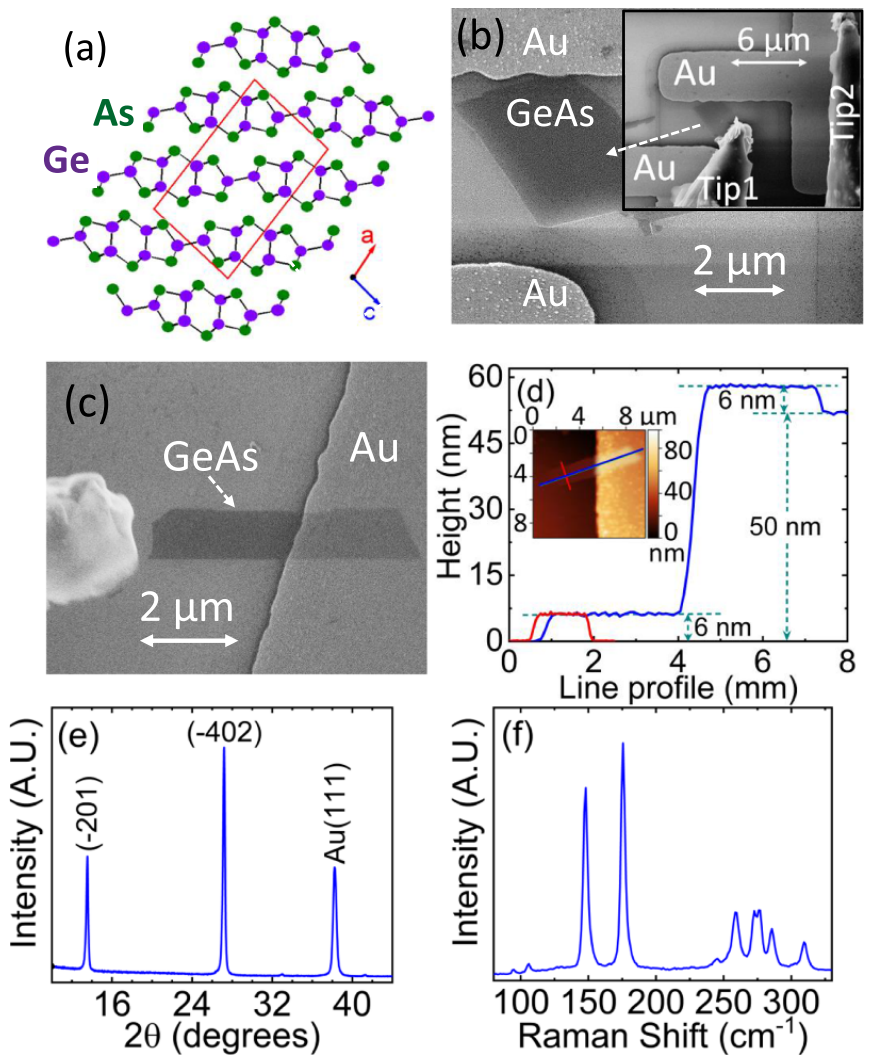

Figure 1. (a) Side view for the crystal structure of layered GeAs. (b) SEM image showing a GeAs flake in tiny contact with the $\mathrm{Au}$ electrode (top marker). The inset shows the same flake contacted by a tungsten tip (Tip 1); a second tungsten tip (Tip 2) is on the Au marker. The Au marker is used as the cathode while Tip 1 is used as the anode in the electrical measurements. (c) SEM image of another GeAs flake used for field emission measurements, with anode tip at hundreds $\mathrm{nm}$ from the flake. (d) AFM profile of the GeAs flake of figure (c), showing the nanosheet thickness of $6 \mathrm{~nm}$. (e) XRD pattern in Bragg-Brentano geometry and (f) Raman spectrum of the same GeAs flake, measured in the parallel configuration.

with the Au marker. The contact with the Au marker is wider for the flake of figure 1(c), which has a uniform thickness of about $6 \mathrm{~nm}$, corresponding to about ten monolayers, as shown in figure 1(d) by its height profile as measured by atomic force microscopy (AFM).

The electrical conduction of the GeAs flakes was measured inside the SEM chamber at pressure below $10^{-6}$ Torr and at room temperature. The flakes were gently contacted by means of two piezo-driven tungsten nanotips (Tip 1 and Tip 2) with nanometric movement control, used as the anode and the cathode, respectively. Such tips were connected to a semiconductor parameter analyzer Keithley 4200-SCS, used as sourcemeter unit. To take advantage of the usually wider and more stable electrical contact produced by the van de Waals force between the GeAs nanosheet and the Au marker, we often chose flakes in electrical contact with a marker. In this configuration, the Au marker was used as the cathode and the tungsten tip in direct contact with the flake was used as the anode (inset of figure 1(b)). The Si substrate offered a third terminal and was used as the gate in a three-probe FET configuration. 
A simple variation of this setup, with the tungsten tip (anode) detached from the flake and positioned at a fixed distance from the GeAs nanosheet (figure 1(c)) allowed the measurement of the local FE current from the nanoflake.

The X-ray diffraction (XRD) was performed by means of a Bruker D5000 system equipped with $\mathrm{Cu} K \alpha$ (wavelength $\lambda=0.154 \mathrm{~nm}$ ) line excitation source. The patterns were acquired in Bragg-Brentano mode that enables measurements of the crystal orientation of the GeAs flakes with respect to the substrate surface as well as of their lattice parameters. Raman spectroscopy measurements were performed using a LabRam high-resolution Micro Raman apparatus by Jobin Yvon with $\lambda_{0}=632 \mathrm{~nm}$ excitation.

\section{Results and discussion}

\subsection{Structural and crystal symmetries characterization}

Figure 1(e) reports the XRD pattern of the sample that we used to determine the unit cell parameters by Rietveld refinement. The peaks identified in figure 1(e) correspond to the $(-201)$ and $(-402)$ base-centered monoclinic structure of GeAs, and the (111) planes of $\mathrm{Au}$ from the Au markers. The structure of GeAs was solved in the monoclinic space group $C 2 / \mathrm{m}$ (12) (JCPDS 011-0524) and the obtained lattice parameters are: $a=1.5552 \mathrm{~nm}, b=0.3761 \mathrm{~nm}, c=0.9524 \mathrm{~nm}$ and $\beta_{\widehat{a c}}=101.255^{\circ}$. Furthermore, the XRD spectrum confirms an interlayer spacing of $0.66 \mathrm{~nm}$ in agreement with previously reported data [20]. These observations lead to the ball and stick model of figure 1(a), showing the GeAs crystal structure in the projection plane (010) along with the cell edges (red line). In figure 1(a), two different geometric orientations of the $\mathrm{Ge}-\mathrm{Ge}$ bond can be clearly distinguished from the crystalline structure of the GeAs: one bond parallel and the other one perpendicular to the layer plane that is an evidence of the anisotropic nature of GeAs crystal structure. The Raman spectrum shown in figure 1(f) evidences multiple Raman active modes peaks due to highly asymmetric structure, with eight of them being $A_{\mathrm{g}}$ modes $\left(95 \mathrm{~cm}^{-1}, 106 \mathrm{~cm}^{-1}, 148 \mathrm{~cm}^{-1}, 175 \mathrm{~cm}^{-1}\right.$, $273 \mathrm{~cm}^{-1}, 276 \mathrm{~cm}^{-1}, 285 \mathrm{~cm}^{-1}$ and $\left.309 \mathrm{~cm}^{-1}\right)$, and one being $B_{\mathrm{g}}$ mode $\left(259 \mathrm{~cm}^{-1}\right)$. XRD and Raman measurements confirm that the GeAs flakes, exfoliated from crystal and transferred onto the substrate, retained the structural properties of the parent crystal also after the deposition.

The electrical conductance of the GeAs nanoflake of figure 1(b), used as the channel of a back-gate FET, was measured in the common-source configuration schematically displayed in figure 2(a). The output characteristics, i.e. $I_{\mathrm{d}}-$ $V_{\text {ds }}$ curves for fixed $V_{\mathrm{gs}}$, of figure 2(b) show that the channel current is modulated by the gate and increases for negative $V_{\mathrm{gs}}$, a behavior that is typical of a channel with hole conduction and has been seen already in similar devices $[5,6]$. The non-linearity of $I_{\mathrm{d}}-V_{\mathrm{ds}}$ curves can be explained considering that the anode and cathode contacts are realized with different metals and have different areas. The $I_{\mathrm{d}}-V_{\mathrm{ds}}$ asymmetric behavior is indicative of slightly different Schottky barriers formed at the W(tip)-GeAs and Au/GeAs interfaces [21, 22]. The
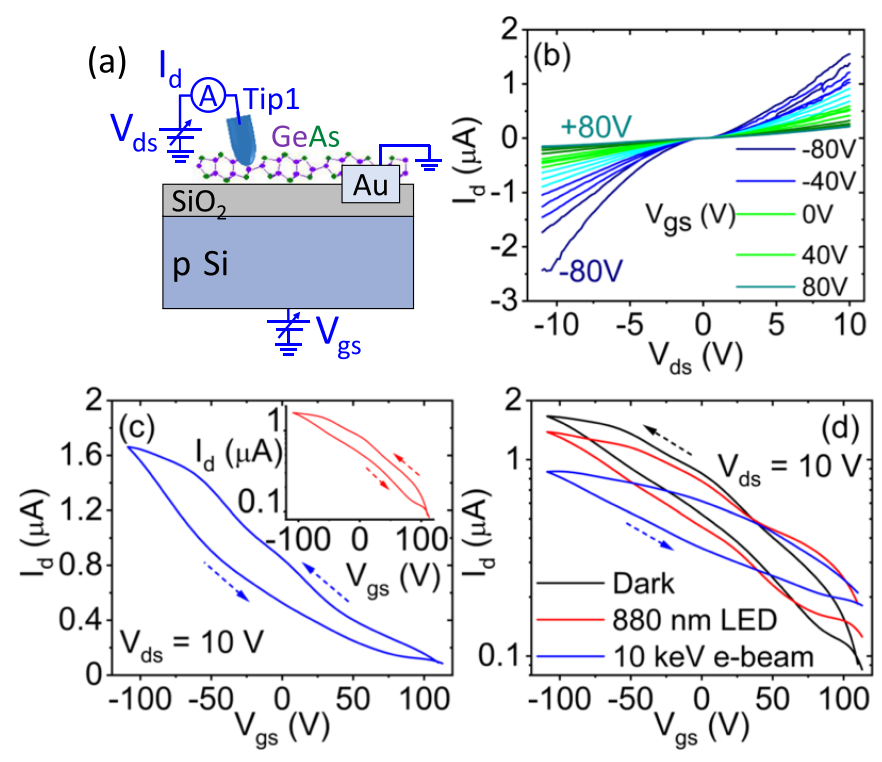

Figure 2. (a) Schematic of the back-gate transistor used for the electrical characterization of the GeAs nanosheets. Electrical characterization of the nanoflake of figure 1(b): (b) output characteristics $I_{\mathrm{d}}-V_{\mathrm{ds}}$ for stepping $V_{\mathrm{gs}}$, showing gate current modulation; (c) transfer characteristics $I_{\mathrm{d}}-V_{\mathrm{gs}}$ for a fixed $V_{\mathrm{ds}}$ with the current on linear and logarithmic scale (inset); (d) transfer characteristics in dark, under $880 \mathrm{~nm}-265 \mu \mathrm{W} \mathrm{cm}{ }^{-2}$ LED light, and after $10 \mathrm{keV}-10 \mathrm{pA}$ electron (e-beam) irradiation. All

measurements are performed at low pressure below $10^{-6}$ Torr and at room temperature.

p-type conduction of the GeAs nanoflake is confirmed by figure 2(c), reporting the transfer characteristic, $I_{\mathrm{d}}-V_{\mathrm{gs}}$ curve for a fixed $V_{\mathrm{ds}}$, and showing that the transistor current decreases for increasing $V_{\mathrm{gs}}$. The modulation of the current is limited to one order of magnitude, despite the quite large $V_{\mathrm{gs}}$ range, consistently with the multilayer nature of the GeAs flake corresponding to a bandgap around several hundred meV. From the slope of the transfer characteristic, we estimated the fieldeffect mobility as $\mu=\frac{L}{W C_{\mathrm{ox}} V_{\mathrm{ds}}} \frac{\mathrm{d} I_{\mathrm{d}}}{\mathrm{d} V_{\mathrm{gs}}} \approx 0.6 \mathrm{~cm}^{2} V^{-1} s^{-1}$, where $C_{\text {ox }}=11.5 \mathrm{nFcm}^{-2}$ is the $\mathrm{SiO}_{2}$ capacitance per unit area, $L \sim 2 \mu \mathrm{m}$ and $\mathrm{W} \sim 0.5 \mu \mathrm{m}$ are the channel length and width, roughly measured from the SEM images. Such mobility is at the low side of the range reported in the literature $[3,5,7]$ being likely affected by the high contact resistance [23]. We observe that the low current as well as the low mobility point to electrical conduction mainly along the armchair direction [5, 6]. Figure 2(c) shows clockwise hysteresis between the forward and reverse sweeps. Hysteresis in the transfer characteristics is a well-known feature of FETs with 2D-material channels and can be caused by gate-induced trapping/detrapping of free carriers in gap states [24-27]. Gap states are caused by external impurities and structural defects or are induced by surface adsorbates and interaction with the gate dielectric. However, electrical measurements were performed in a high vacuum, and adsorbates like water or oxygen should play a minor role while gap trap states from impurities [6] or $\mathrm{GeAs} / \mathrm{SiO}_{2}$ interaction should dominate. 
Light or electron beam irradiation [28, 29] can strongly affect 2D-FETs. Their impact on GeAs is shown in figure 2(d). Exposure to either $880 \mathrm{~nm}-265 \mu \mathrm{Wcm}^{-2}$ LED light or $10 \mathrm{keV}-10 \mathrm{pA} e$-beam results in a reduction (increase) of the current in the on (off) state, making the gate control weaker. This behavior corresponds to negative 'photo'-conductivity, as reported for some other nanomaterials. Negative photoconductivity has been observed in InAs nanowire transistors [30] or in carbon nanotube films [31] exposed to air, $\mathrm{H}_{2} \mathrm{O}$ and $\mathrm{O}_{2}$ atmospheres. It has been attributed to photodesorption of water molecules and photo-assisted chemisorption of $\mathrm{O}_{2}$ molecules [30] as well as to photogating effect due to the excitation of photogenerated electrons into the native oxide layer covering the nanowires [30]. Negative photoconductivity has been also reported in thick $(0.32 \mathrm{~mm})$ tablets of copper selenide $\left(\mathrm{Cu}_{2-x} \mathrm{Se}\right)$ powders and explained by a photothermal effect leading to charge carrier depletion from the channel region under the influence of a temperature gradient [32]. We expect a marginal role of $\mathrm{H}_{2} \mathrm{O}$ and $\mathrm{O}_{2}$ molecules for the GeAs flakes under study because we performed the measurements after keeping the samples in a high vacuum for a few days; similarly, the photothermal effect is excluded by the ultralow thickness. Therefore, the effect that most likely causes the negative photoconductivity is the photogating effect that overtakes the photoconductive effect in GeAs at $V_{\mathrm{gs}}<0 \mathrm{~V}[33,34]$. This effect arises from the interaction of light or electron beam that generate electron-hole pairs in the GeAs and the $\mathrm{SiO}_{2} / \mathrm{Si}$ substrate. The vertical band bending and the negative $V_{\mathrm{gs}}$ favor the accumulation of holes in the $\mathrm{SiO}_{2}$ layer below the channel as well as in intrinsic defects of GeAs [4]. Such trapped holes add a positive gate voltage that decreases the p-type channel conductance (the opposite occurs at $V_{\mathrm{gs}}>0 \mathrm{~V}$ when photogenerated electrons are attracted at the $\mathrm{GeAs} / \mathrm{SiO}_{2}$ interface causing an increase of the current).

Although they are regulated by a similar mechanism, it can be observed that the greatest reduction in conductivity occurs under $e$-beam irradiation. This is due to the power density per area $\left(\sim 1 \mathrm{Wcm}^{-2}\right)$ of the $10 \mathrm{keV}-10 \mathrm{pA}$ electron beam that is considerably higher than the power of the $880 \mathrm{~nm}$ LED $\left(-265 \mu \mathrm{Wcm}^{-2}\right)$.

\subsection{Field emission}

The $I_{\mathrm{d}}-V_{\mathrm{ds}}$ output curves shown in figure 3(a) were measured with Tip 1 in direct contact with the flake of figure 1(c) and Tip 2 on the Au marker, while the gate was grounded; the curves confirm the nonlinear p-type behavior of the GeAs nanoflakes as well as their negative photoconduction, as evidenced by the reduced current under $880 \mathrm{~nm}$ LED illumination. After the $I_{\mathrm{ds}}-V_{\mathrm{ds}}$ measurements, Tip 1 was detached from the flake and brought at about $400 \mathrm{~nm}$ from its edge (see figure 1(c) and inset of figure 3(b)). With the gate grounded, the voltage of Tip 1 (the anode) was slowly increased up to $110 \mathrm{~V}$, while monitoring the current. Figure 3(b) shows that the current remains at the noise floor of the experimental setup up to $\sim 65 \mathrm{~V}$, then it increases exponentially for more than three orders of
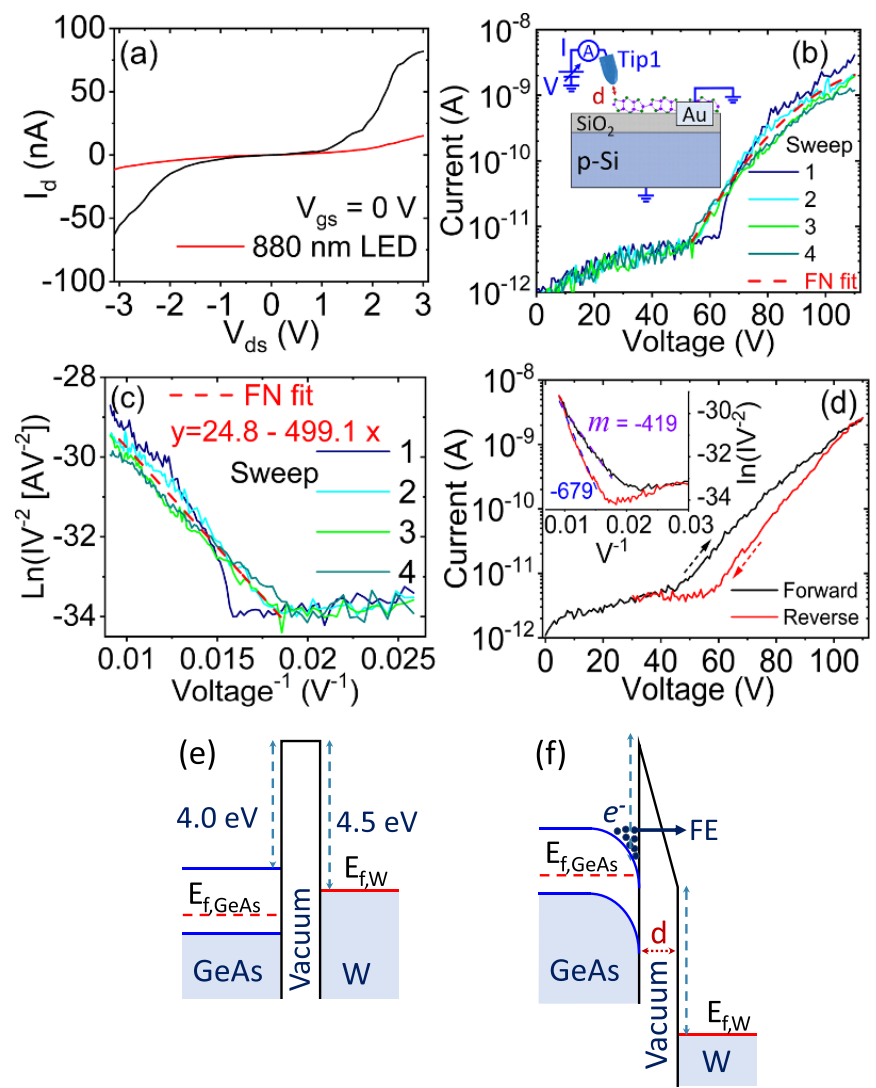

Figure 3. Electrical characterization of the GeAs nanoflake of figure 1(c). (a) Output characteristics $I_{\mathrm{d}}-V_{\mathrm{ds}}$ at $V_{\mathrm{gs}}=0 \mathrm{~V}$, in dark and under $880 \mathrm{~nm}$ LED illumination with Tip 1 in contact with the flake. (b) Current-voltage measurements with Tip 1 at $d=400 \mathrm{~nm}$ from the edge of the flake showing a stable field emission current for $V>50 \mathrm{~V}$. (c) Fowler-Nordheim plot. (d) Current-voltage measurements and Fowler-Nordheim plot (inset) for forward and backward Tip 1 voltage swept. Band diagram of the GeAs/vacuum/Tip 1 system without (e) and with (f) positive bias applied to Tip 1 .

magnitude. The behavior of the current is typical of FE. The phenomenon is reproducible, with the FE current turning on at a slightly lower voltage $(\sim 52 \mathrm{~V})$ for the successive anode voltage sweeps. The small reduction of the current with respect to the first sweep is likely due to an electrical conditioning effect, resulting in the desorption of residues or adsorbates at the edge of the flake by Joule heating during the first sweep.

A FE theory to describe the current trend as function of the applied voltage was firstly developed by Fowler-Nordheim (FN), which considered electron tunneling from a flat surface through a triangular barrier and relies on Sommerfeld's freeelectron theory for the description of the electronic energy distribution. Modern nano-structured devices sometimes require the use of more complex equations to describe the FE current behavior from rough, irregular surfaces [35, 36]. However, it has been repeatedly shown that in many cases the FN theory [37] is sufficient for the analysis of FE curves from 2D materials providing reliable estimations of the typical figures of merit that are used in the evaluation of the emitter. According to it, 
the FE current can be expressed as:

$$
I=S \cdot A \phi^{-1}\left(\beta \frac{V}{k d}\right)^{2} \exp \left[-B \phi^{3 / 2}\left(\beta \frac{V}{k d}\right)^{-1}\right]
$$

where $S$ is the emitting area, $\mathrm{A}=1.54 \times 10^{-6} \mathrm{AV}^{-2} \mathrm{eV}$ and $B=6.83 \times 10^{9} \mathrm{eV}^{-3 / 2} \mathrm{~m}^{-1} \mathrm{~V}$ are dimensional constants, $\phi$ is the material work function, $V / k d=E$ is the electric field due to the applied voltage $\mathrm{V}$ when the anode-cathode separation distance is $d=400 \mathrm{~nm}$ with $k \sim 1.6$ a phenomenological factor accounting for the spherical shape of the anode [38]. $\beta$ represents the so-called field enhancement factor due to the accumulation of the electrical field lines on the sharper protrusions of the emitting surface [39]. Such protrusions are the main emitting sites and in the present application correspond to the edge of the nanoflake. The FN equation (1) well fits the experimental data, as shown by the red dashed curve in figure 3(b). The so-called FN plot of $\ln \left(I V^{-2}\right)$ vs $V^{-1}$, shown in figure 3(c), further corroborates the FN nature of the observed current.

Figure 3(d) displays the FE current and the FN plot (inset) over a forward and reverse sweep. Interestingly, the FE current decreases during the reverse sweep. This hysteretic behavior could arise from space charge, which suppresses the emission during the ramp down of the anodic voltage.

The FN plot allows to estimate the field enhancement factor as $\beta=-B k d \phi^{3 / 2} \mathrm{~m}^{-1}$, where $\mathrm{m}$ is the slope of the fitting (dashed red) straight line. Assuming $\phi=4.0 \mathrm{eV}$ for multilayer GeAs, $\beta \sim 70$, according to the measurements of figure 3(c).

The turn-on field, defined as the electric field to extract the current of $10 \mathrm{pA}$, is $E_{\mathrm{to}} \sim 80 \mathrm{~V} \mu \mathrm{m}^{-1}$. This result is comparable to those usually obtained for other non-engineered $2 \mathrm{D}$ materials. Turn-on field can be further improved by designing an appropriately shaped device, for example with a sharp termination that enhances the local electric field and favors the extraction of electrons. The p-type doping of GeAs contributes to increase $E_{\mathrm{to}}$. Indeed, extraction of electrons from a p-type materials requires the achievement of an inversion condition at the emitting surface. Such an inversion occurs when the anode voltage is high enough to induce the required band bending, as shown in the energy band diagrams of figures 3(e) and (f). The inversion layer at the GeAs interface provides the electrons to tunnel through the vacuum barrier at high bias.

We finally note that also the $\beta$ factor obtained for GeAs nanoflakes is competitive when compared with those measured in similar experimental conditions from other 2D materials such as mono and multilayers graphene, $\mathrm{WSe}_{2}$ and $\mathrm{PdSe}_{2}$ [12, 17, 40-43]. Furthermore, conservatively assuming that the emission occurs from the entire edge of the flake, the extracted current density attains the appreciable value of $\sim 10 \mathrm{~A} \mathrm{~cm}^{-2}$. This remarkable result, together with the reported high reproducibility, makes the GeAs a field emitter capable of competing with established field emitters, known for their capability to reach high current densities without degradation. Indeed, the FE figures of merit of GeAs result comparable to the FE performance of carbon nanotubes [44-47], Mo tips [48], bare and metal coated Si tips [49], or other established emitter materials [50-55].

\section{Conclusion}

In conclusion, mechanically exfoliated multilayer GeAs nanosheets, transferred onto $\mathrm{SiO}_{2} / \mathrm{Si}$ substrates, have been used as the channel of back gated transistors and as cold cathodes for electron emission. It has been found that the nanosheets possess intrinsic p-type doping conductivity modulated by the gate, mobility of $0.6 \mathrm{~cm}^{2} \mathrm{~V}^{-1} \mathrm{~s}^{-1}$, and negative photoconductivity. A reproducible FE current from the edge of GeAs nanoflakes occurring with a turn-on field around

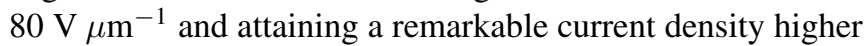
than $10 \mathrm{~A} \mathrm{~cm}^{-2}$ has been reported.

This study provides experimental evidence of FE from GeAs nanoflakes and paves the way for new applications of 2D GeAs introducing it to the realm of vacuum electronics.

\section{Acknowledgments}

This research was funded by the Italian Ministry of Education, University and Research (MIUR), projects Pico \& Pro ARS01_01061 and RINASCIMENTO ARS01_01088. $\mathrm{L} \mathrm{C}$ and $\mathrm{J} \mathrm{S}$ want to express their gratitude to the Villum Fonden (Young Investigator Program, Project No. 19130). L C acknowledges support from MIUR via 'Programma per Giovani Ricercatori-Rita Levi Montalcini 2017’.

\section{ORCID iDs}

Antonio Di Bartolomeo (D) https://orcid.org/0000-00023629-726X

Alessandro Grillo (D) https://orcid.org/0000-0002-8909-9865

Filippo Giubileo (D) https://orcid.org/0000-0003-2233-3810

Luca Camilli (D) https://orcid.org/0000-0003-2498-0210

Jianbo Sun (D) https://orcid.org/0000-0001-9741-8726

Maurizio Passacantando (D) https://orcid.org/0000-00023680-5295

\section{References}

[1] Wei Y, Fang L, Tong X and Liu R 2019 First principles study on the two-dimensional germanium arsenide containing vacancy Sci. Discov. 7188

[2] Lee K, Kamali S, Ericsson T, Bellard M and Kovnir K 2016 GeAs: highly anisotropic van der Waals thermoelectric material Chem. Mater. 28 2776-85

[3] Jung C S, Kim D, Cha S, Myung Y, Shojaei F, Abbas H G, Lee J A, Cha E H, Park J and Kang H S 2018 Two-dimensional GeAs with a visible range band gap $J$. Mater. Chem. A 6 9089-98

[4] Zhou L, Guo Y and Zhao J 2018 GeAs and SiAs monolayers: novel 2D semiconductors with suitable band structures Physica E 95 149-53

[5] Grillo A, Di Bartolomeo A, Urban F, Passacantando M, Caridad J M, Sun J and Camilli L 2020 Observation of 2D conduction in ultrathin germanium arsenide field-effect transistors ACS Appl. Mater. Interfaces 12 12998-3004

[6] Sun J, Passacantando M, Palummo M, Nardone M, Kaasbjerg K, Grillo A, Di Bartolomeo A, Caridad J M and Camilli L 2020 Impact of impurities on the electrical 
conduction of anisotropic two-dimensional materials Phys. Rev. Appl. 13044063

[7] Yang S, Yang Y, Wu M, Hu C, Shen W, Gong Y, Huang L, Jiang C, Zhang Y and Ajayan P M 2018 Highly in-plane optical and electrical anisotropy of 2D germanium arsenide Adv. Funct. Mater. 281707379

[8] Mortazavi B and Rabczuk T 2018 Anisotropic mechanical properties and strain tuneable band-gap in single-layer SiP, SiAs, GeP and GeAs Physica E 103 273-8

[9] Zhou Z et al 2018 Perpendicular optical reversal of the linear dichroism and polarized photodetection in $2 \mathrm{D}$ GeAs ACS Nano 12 12416-23

[10] Mortazavi B, Shahrokhi M, Cuniberti G and Zhuang X 2019 Two-dimensional SiP, SiAs, GeP and GeAs as promising candidates for photocatalytic applications Coatings 9522

[11] Passacantando M, Bussolotti F, Santucci S, Di Bartolomeo A, Giubileo F, Iemmo L and Cucolo A M 2008 Field emission from a selected multiwall carbon nanotube Nanotechnology 19395701

[12] Santandrea S, Giubileo F, Grossi V, Santucci S, Passacantando M, Schroeder T, Lupina G and Di Bartolomeo A 2011 Field emission from single and few-layer graphene flakes Appl. Phys. Lett. 98163109

[13] Robertson J 2002 Field emission applications of carbon nanotubes AIP Conf. Proc. 633 537-42

[14] Giubileo F, Bartolomeo A D, Scarfato A, Iemmo L, Bobba F, Passacantando M, Santucci S and Cucolo A M 2009 Local probing of the field emission stability of vertically aligned multi-walled carbon nanotubes Carbon 47 1074-80

[15] Schreiner R et al 2015. Semiconductor field emission electron sources using a modular system concept for application in sensors and X-ray-sources 2015: 28th Int. Vacuum Nanoelectronics Conf. (IVNC) (Guangzhou, China: IEEE) pp 178-9

[16] Giubileo F, Di Bartolomeo A, Iemmo L, Luongo G and Urban F 2018 Field emission from carbon nanostructures Appl. Sci. 8526

[17] Di Bartolomeo A, Urban F, Passacantando M, McEvoy N, Peters L, Iemmo L, Luongo G, Romeo F and Giubileo F 2019 A WSe2 vertical field emission transistor Nanoscale 11 1538-48

[18] Nirantar S, Ahmed T, Bhaskaran M, Han J-W, Walia S and Sriram S 2019 Electron emission devices for energy-efficient systems Adv. Intell. Syst. 11900039

[19] Di Bartolomeo A, Passacantando M, Niu G, Schlykow V, Lupina G, Giubileo F and Schroeder T 2016 Observation of field emission from GeSn nanoparticles epitaxially grown on silicon nanopillar arrays Nanotechnology 27485707

[20] Sun J, Giorgi G, Palummo M, Sutter P, Passacantando M and Camilli L 2020 A scalable method for thickness and lateral engineering of 2D materials ACS Nano 14 4861-70

[21] Di Bartolomeo A et al 2018 Asymmetric Schottky contacts in bilayer $\mathrm{MoS}_{2}$ field effect transistors Adv. Funct. Mater. 281800657

[22] Di Bartolomeo A, Giubileo F, Grillo A, Luongo G, Iemmo L, Urban F, Lozzi L, Capista D, Nardone M and Passacantando M 2019 Bias tunable photocurrent in metal-insulator-semiconductor heterostructures with photoresponse enhanced by carbon nanotubes Nanomaterials 91598

[23] Urban F, Lupina G, Grillo A, Martucciello N and Di Bartolomeo A 2020 Contact resistance and mobility in back-gate graphene transistors Nano Express 1010001

[24] Late D J, Liu B, Matte H S S R, Dravid V P and Rao C N R 2012 Hysteresis in single-layer $\mathrm{MoS}_{2}$ field effect transistors ACS Nano 6 5635-41

[25] Di Bartolomeo A, Genovese L, Giubileo F, Iemmo L, Luongo G, Foller T and Schleberger M 2017 Hysteresis in the transfer characteristics of $\mathrm{MoS}_{2}$ transistors $2 \mathrm{D}$ Mater. 5015014

[26] Kaushik N, Mackenzie D M A, Thakar K, Goyal N, Mukherjee B, Boggild P, Petersen D H and Lodha S 2017 Reversible hysteresis inversion in $\mathrm{MoS}_{2}$ field effect transistors Npj 2D Mater. Appl. 134

[27] Di Bartolomeo A, Pelella A, Liu X, Miao F, Passacantando M, Giubileo F, Grillo A, Iemmo L, Urban F and Liang S 2019 Pressure-tunable ambipolar conduction and hysteresis in thin palladium diselenide field effect transistors Adv. Funct. Mater. 291902483

[28] Giubileo F, Iemmo L, Passacantando M, Urban F, Luongo G, Sun L, Amato G, Enrico E and Di Bartolomeo A 2019 Effect of electron irradiation on the transport and field emission properties of few-layer $\mathrm{MoS}_{2}$ field-effect transistors J. Phys. Chem. C 123 1454-61

[29] Di Bartolomeo A, Urban F, Pelella A, Grillo A, Passacantando M, Liu X and Giubileo F 2020 Electron irradiation of multilayer $\mathrm{PdSe}_{2}$ field effect transistors Nanotechnology 31375204

[30] Han Y, Zheng X, Fu M, Pan D, Li X, Guo Y, Zhao J and Chen Q 2016 Negative photoconductivity of InAs nanowires Phys. Chem. Chem. Phys. 18 818-26

[31] Zhu J-L, Zhang G, Wei J and Sun J-L 2012 Negative and positive photoconductivity modulated by light wavelengths in carbon nanotube film Appl. Phys. Lett. 101123117

[32] Singh S C, Peng Y, Rutledge J and Guo C 2019 Photothermal and Joule-heating-induced negative-photoconductivitybased ultraresponsive and near-zero-biased copper selenide photodetectors ACS Appl. Electron. Mater. 1 1169-78

[33] Furchi M M, Polyushkin D K, Pospischil A and Mueller T 2014 Mechanisms of photoconductivity in atomically thin $\mathrm{MoS}_{2}$ Nano Lett. 14 6165-70

[34] Di Bartolomeo A, Genovese L, Foller T, Giubileo F, Luongo G, Croin L, Liang S-J, Ang L K and Schleberger M 2017 Electrical transport and persistent photoconductivity in monolayer $\mathrm{MoS}_{2}$ phototransistors Nanotechnology 28214002

[35] Yuasa K, Shimoi A, Ohba I and Oshima C 2002 Modified Fowler-Nordheim field emission formulae from a nonplanar emitter model Surf. Sci. 520 18-28

[36] Zubair M, Ang Y S and Ang L K 2018 Fractional Fowler-Nordheim law for field emission from rough surface with nonparabolic energy dispersion IEEE Trans. Electron Devices 65 2089-95

[37] Anon 2017 Fowler-Nordheim equation Introduction to the Physics of Electron Emission (Chichester, UK: Wiley) pp 139-48

[38] Di Bartolomeo A, Scarfato A, Giubileo F, Bobba F, Biasiucci M, Cucolo A M, Santucci S and Passacantando M 2007 A local field emission study of partially aligned carbon-nanotubes by atomic force microscope probe Carbon 45 2957-71

[39] Giubileo F, Passacantando M, Urban F, Grillo A, Iemmo L, Pelella A, Goosney C, LaPierre R and Di Bartolomeo A 2020 Field emission characteristics of InSb patterned nanowires Adv. Electron. Mater. 62000402

[40] Di Bartolomeo A, Giubileo F, Iemmo L, Romeo F, Russo S, Unal S, Passacantando M, Grossi V and Cucolo A M 2016 Leakage and field emission in side-gate graphene field effect transistors Appl. Phys. Lett. 109023510

[41] Urban F, Passacantando M, Giubileo F, Iemmo L and Di Bartolomeo A 2018 Transport and field emission properties of $\mathrm{MoS}_{2}$ bilayers Nanomaterials 8151

[42] Iemmo L, Urban F, Giubileo F, Passacantando M and Di Bartolomeo A 2020 Nanotip contacts for electric transport 
and field emission characterization of ultrathin $\mathrm{MoS}_{2}$ flakes Nanomaterials 10106

[43] Di Bartolomeo A, Pelella A, Urban F, Grillo A, Iemmo L, Passacantando M, Liu X and Giubileo F 2020 Field emission in ultrathin $\mathrm{PdSe}_{2}$ back-gated transistors $A d v$. Electron. Mater. 62000094

[44] Calderón-Colón X, Geng H, Gao B, An L, Cao G and Zhou O 2009 A carbon nanotube field emission cathode with high current density and long-term stability Nanotechnology 20325707

[45] Giubileo F, Di Bartolomeo A, Sarno M, Altavilla C, Santandrea S, Ciambelli P and Cucolo A M 2012 Field emission properties of as-grown multiwalled carbon nanotube films Carbon 50 163-9

[46] Zhu W, Bower C, Zhou O, Kochanski G and Jin S 1999 Very large current density from carbon nanotube field emitters Int. Electron Devices Meeting 1999. Technical Digest (Cat. No.99CH36318) (Washington, DC: IEEE) pp 705-8

[47] Zhang Q, Wang X, Meng P, Yue H, Zheng R, Wu X and Cheng G 2018 High current density and low emission field of carbon nanotube array microbundle Appl. Phys. Lett. 112013101

[48] Lin C M, Chang S J, Yokoyama M, Lin I-N, Chen J F and Huang B R 2000 Field-emission enhancement of Mo-tip field-emitted arrays fabricated by using a redox method IEEE Electron Device Lett. 21 560-2
[49] Günther B, Kaldasch F, Müller G, Schmitt S, Henning T, Huber R and Lacher M 2003 Uniformity and stability of field emission from bare and metal coated Si tip arrays $J$. Vac. Sci. Technol. B 21427

[50] Grillo A, Barrat J, Galazka Z, Passacantando M, Giubileo F, Iemmo L, Luongo G, Urban F, Dubourdieu C and Di Bartolomeo A 2019 High field-emission current density from $\beta$-Ga2O3 nanopillars Appl. Phys. Lett. 114193101

[51] Giubileo F, Di Bartolomeo A, Iemmo L, Luongo G, Passacantando M, Koivusalo E, Hakkarainen T and Guina M 2017 Field emission from self-catalyzed GaAs nanowires Nanomaterials 7275

[52] Lv S, Li Z, Liao J, Wang G, Li M and Miao W 2015 Optimizing field emission properties of the hybrid structures of graphene stretched on patterned and size-controllable SiNWs Sci. Rep. 515035

[53] Iemmo L, Di Bartolomeo A, Giubileo F, Luongo G, Passacantando M, Niu G, Hatami F, Skibitzki O and Schroeder T 2017 Graphene enhanced field emission from InP nanocrystals Nanotechnology 28495705

[54] Giubileo F, Grillo A, Passacantando M, Urban F, Iemmo L, Luongo G, Pelella A, Loveridge M, Lozzi L and Di Bartolomeo A 2019 Field emission characterization of $\mathrm{MoS}_{2}$ nanoflowers Nanomaterials 9717

[55] Nguyen H D, Kang J S, Li M and Hu Y 2019 High-performance field emission based on nanostructured tin selenide for nanoscale vacuum transistors Nanoscale 11 3129-37 